\title{
Acetylome in Human Fibroblasts From Parkinson's Disease Patients
}

\author{
Sokhna M. S. Yakhine-Diop ${ }^{1,2}$, Mario Rodríguez-Arribas ${ }^{1,2}$, Guadalupe Martínez-Chacón ${ }^{1,2}$, \\ Elisabet Uribe-Carretero ${ }^{1,2}$, Rubén Gómez-Sánchez ${ }^{3}$, Ana Aiastui ${ }^{1,4,5}$, \\ Adolfo López de Munain 1,5,6,7,8, José M. Bravo-San Pedro 9,10,11,12,13, Mireia Niso-Santano ${ }^{1,2}$, \\ Rosa A. González-Polo ${ }^{1,2 *}$ and José M. Fuentes ${ }^{1,2 *}$
}

\begin{abstract}
${ }^{1}$ Centro de Investigación Biomédica en Red en Enfermedades Neurodegenerativas, Madrid, Spain, ${ }^{2}$ Departamento de Bioquímica y Biología Molecular y Genética, Facultad de Enfermería y Terapia Ocupacional, Universidad de Extremadura, Cáceres, Spain, ${ }^{3}$ Department of Cell Biology, University of Groningen, University Medical Center Groningen, Groningen, Netherlands, ${ }^{4}$ Cell Culture Plataform, Donostia University Hospital, San Sebastián, Spain, ${ }^{5}$ Neuroscience Area of Biodonostia Health Research Institute, Donostia University Hospital, San Sebastián, Spain, ${ }^{6}$ Department of Neurology, Donostia University Hospital, San Sebastian, Spain, ${ }^{7} / l u n d a i n$ Fundazioa, San Sebastian, Spain, ${ }^{8}$ Department of Neurosciences, University of the Basque Country UPV-EHU, San Sebastián, Spain, ${ }^{9}$ Equipe 11 labellisèe Ligue Contre le Cancer, Centre de Recherche des Cordeliers, Paris, France, ${ }^{10}$ INSERM U1138, Paris, France, ${ }^{11}$ Université Paris Descartes/Paris V, Sorbonne Paris Cité, Paris, France, ${ }^{12}$ Université Pierre et Marie Curie/Paris VI, Paris, France, ${ }^{13}$ Gustave Roussy Comprehensive Cancer Institute, Villejuif, France
\end{abstract}

\section{OPEN ACCESS}

Edited by:

Merce Pallas,

Universitat de Barcelona, Spain

Reviewed by:

Joaquin Jordan,

Universidad de Castilla La Mancha

Albacete, Spain

Carlos Guillén,

Complutense University of Madrid,

Spain

*Correspondence:

Rosa A. González-Polo rosapolo@unex.es José M. Fuentes jfuentes@unex.es

Received: 30 January 2018 Accepted: 22 March 2018 Published: 17 April 2018

Citation:

Yakhine-Diop SMS Rodríguez-Arribas $M$, Martínez-Chacón G,

Uribe-Carretero E, Gómez-Sánchez $R$, Aiastui A, López de Munain A, Bravo-San Pedro JM, Niso-Santano M, González-Polo RA and Fuentes JM (2018) Acetylome in Human Fibroblasts From Parkinson's

Disease Patients.

Front. Cell. Neurosci. 12:97. doi: 10.3389/fncel.2018.00097
Parkinson's disease (PD) is a multifactorial neurodegenerative disorder. The pathogenesis of this disease is associated with gene and environmental factors. Mutations in leucine-rich repeat kinase 2 (LRRK2) are the most frequent genetic cause of familial and sporadic PD. Moreover, posttranslational modifications, including protein acetylation, are involved in the molecular mechanism of PD. Acetylation of lysine proteins is a dynamic process that is modulated in PD. In this descriptive study, we characterized the acetylated proteins and peptides in primary fibroblasts from idiopathic PD (IPD) and genetic PD harboring G2019S or R1441G LRRK2 mutations. Identified acetylated peptides are modulated between individuals' groups. Although acetylated nuclear proteins are the most represented in cells, they are hypoacetylated in IPD. Results display that the level of hyperacetylated and hypoacetylated peptides are, respectively, enhanced in genetic PD and in IPD cells.

Keywords: acetylation, LRRK2, peptides, Parkinson, proteins

\section{INTRODUCTION}

Parkinson's disease (PD) is a disabling neurological disorder that is in progressive evolution. This neurodegeneration mainly affects the dopaminergic neurons of the susbtancia nigra pars compacta that are in part responsible for clinical motor symptoms. The widespread of this neurodegeneration from the midbrain to other neurotransmitter (serotoninergic and noradrenergic) systems elicits the appearance of non-motor symptoms (Politis and Loane, 2011; Deusser et al., 2015). Although well not understood, the etiopathogenesis of PD has thought related to environmental factors and gene mutations. Almost 90\% of PD cases are sporadic (Ammal Kaidery et al., 2013), and may due to the susceptibility of genetic predisposition to environmental factors (Yakhine-Diop et al., 2014). Mutations in leucine-rich repeat kinase 2 (LRRK2) have been associated with autosomal dominant PD and involved in familial and idiopathic cases. Among six pathogenic LRRK2 mutations

Abbreviations: CI, Confidence interval; FA, Trifluoroacetic acid; FDR, False decoy recovery; GAPDH, Glyceraldehyde-3phosphate dehydrogenase; H2B, Histone 2B; HAT, Histone acetyltransferase; HDAC, Histone deacetylase; ID, Identification; IPD, Idiopathic Parkinson's disease; K, Lysine; LC-MS, Liquid chromatography-mass spectrometry; LRRK2, Leucinerich repeat kinase 2; PD, Parkinson's disease; PDHA1, Pyruvate dehydrogenase; PSPEP, Proteomics System Performance Evaluation Pipeline Software; ROS, Reactive oxygen species. 
(R1441G/C/H, Y1699C, G2019S, and I2020T), the most frequent is the G2019S that affect almost 5\% of familial PD and $2 \%$ of sporadic PD (Li et al., 2014). G2019S and R1441G are located, respectively, in the kinase and GTP domains of LRRK2 protein. Both mutations participate in the pathogenicity of PD through the impairment of LRRK2 enzymatic activity (Martin et al., 2014).

Evidence has reported that PARK genes, among other synuclein (SNCA) and LRRK2, regulate epigenetic mechanisms, thereby modulate gene expression (Coppedè, 2012). Indeed, gene expression is altered through posttranslational modifications of histones (methylation, phosphorylation, ubiquitination, and acetylation) and affects individual phenotypes (Ammal Kaidery et al., 2013). Parkinsonism-related toxins modulate histone acetylation by either decreasing histone deacetylase (HDAC) activity (Song et al., 2011) or increasing histone acetyltransferase (HAT) activity (Song et al., 2010). Studies, in PD post mortem brains, have reported that histone acetylation is upregulated in midbrain neurons; however, in some patients, this acetylation level varies differentially according to the brain regions and cell types (Park et al., 2016).

Acetylation is the transfer of an acetyl group from acetyl coenzyme $\mathrm{A}$ to the $\varepsilon$-amino group of lysine residues in proteins (histone and non-histone proteins) or to the $\alpha$-amino group of the $\mathrm{N}$-terminus of proteins. $\mathrm{N}$-terminal acetylation is an irreversible reaction that is catalyzed by $\mathrm{N}$-terminal acetyltransferases, whereas lysine acetylation is regulated by the balance of two enzymes HAT and HDAC (Drazic et al., 2016). The combination of these both acetylation reactions constitutes the acetylome. Acetylome in PD models is poorly characterized, only the variation of histone acetylation has been widely reported. Given that it may have an important role in PD pathogenesis, we identified some of those proteins that are acetylated in PD-associated (G2019S and R1441G) LRRK2 mutations and idiopathic PD. Importantly, there are more acetylated peptides in genetic PD models and acetylated proteins are mainly nuclear and cytosolic.

\section{MATERIALS AND METHODS \\ Cell Culture}

Fibroblasts from PD patients (with or without LRRK2 mutations) and from control subjects were provided by Dr. Adolfo López de Munaín. Experiments were performed using four cell lines: Control (Co, patients who did not develop PD), IPD (IPD, PD patients without LRRK2 mutations), GS (PD patients with G2019S LRRK2 mutation), and RG (PD patients with R1441G LRRK2 mutation). This study was carried out in accordance with the recommendations of Comité Ético de Investigación Clínica del Área Sanitaria de Gipuzkoa. All subjects gave written informed consent in accordance with the Declaration of Helsinki. Cells were grown in Dulbecco's modified Eagle's medium (DMEM, Sigma-Aldrich, D6546) supplemented with $10 \%$ of fetal bovine serum (FBS, SigmaAldrich, F7524), 1\% L-glutamine (Sigma-Aldrich, G7513) and $2 \mathrm{~mL}$ streptomycin/penicillin (HyClone, Thermo Fisher Scientific, SV30010) at $37^{\circ} \mathrm{C}$ in $5 \% \mathrm{CO}_{2} / 95 \%$ air. To confirm the G2019S or R1441G LRRK2 mutations in cells, DNA was extracted
(Macherey-Nagel Kit, 740952.50) and sequenced at STAB VIDA (Caparica, Portugal). In this study, we worked with pooled cell lines ranging from three to four cell lines (Table 1). Human fibroblasts (HFs) were seeded at a density of $3.5 \times 10^{4}$ cells $/ \mathrm{mL}$ and from lower passages.

\section{Digestion and Desalting of Peptides}

Samples (Co, IPD, GS, and RG) were resuspended in $400 \mu \mathrm{L}$ of $50 \mathrm{mM}$ ammoniun bicarbonate and quantified by Bradford protein assay (BioRad). For each sample, $2 \mathrm{mg}$ protein were diluted in $8 \mathrm{M}$ Urea in-solution trypsin digestion. Proteins were reduced, alkylated, and digested with a 1:20 (w/w) ratio of recombinant trypsin sequencing grade (Roche) overnight at $37^{\circ} \mathrm{C}$. Peptides from digested proteins were desalted and concentrated with a $\mathrm{C} 18$ reversed phase chromatography (ZipTip C18, Millipore) and the peptides were eluted in $50 \%$ acetonitrile $(\mathrm{ACN}) / 0.1 \%$ trifluoroacetic acid (FA). Finally, the samples were freeze-dried in SpeedVac and dissolved in $200 \mu \mathrm{L}$ of NETN (100 mM NaCl, $1 \mathrm{mM}$ EDTA, $50 \mathrm{mM}$ Tris $\mathrm{pH}$ 8, and $0.5 \%$ Nonidet P40) buffer for affinity enrichment of lysine-acetylated peptides.

\section{Enrichment of Lysine-Acetylated Peptides}

Samples in NETN buffer were incubated with anti-acetyl lysine agarose beads (catalog no. PTM-104, PTM Biolabs) at $4^{\circ} \mathrm{C}$ overnight with gentle shaking. After incubation, the beads were carefully washed three times with NETN buffer, twice with ETN buffer ( $1 \mathrm{mM}$ EDTA, $50 \mathrm{mM}$ Tris $\mathrm{pH} 8$, and $100 \mathrm{mM} \mathrm{NaCl}$ ), and once with water. The immunoprecipitated peptides were eluted with $1 \%$ FA and dried in a SpeedVac. The resulting peptides were cleaned with C18 Zip Tips (Millipore) according to the manufacturer's instructions and were dissolved in $12 \mu \mathrm{L}$ of $2 \%$ ACN/0.1\% FA then subjected to LC-MS/MS analysis by Triple TOF 5600 (SCIEX).

TABLE 1 | Presentation of the four groups of pooled cell lines.

\begin{tabular}{|c|c|c|c|}
\hline Groups & Names & Dates of Birth & Genotypes \\
\hline & Co1 & & LRRK2 WT \\
\hline \multirow[t]{4}{*}{ Co } & Co2 & 1956-1977 & LRRK2 WT \\
\hline & Co3 & & LRRK2 WT \\
\hline & Co4 & & LRRK2 WT \\
\hline & IPD1 & & LRRK2 WT \\
\hline \multirow[t]{3}{*}{ IPD } & IPD2 & 1928-1954 & LRRK2 WT \\
\hline & IPD3 & & LRRK2 WT \\
\hline & GS1 & & G2019S Heterozygous \\
\hline \multirow[t]{3}{*}{ GS } & GS2 & 1945-1949 & G2019S Heterozygous \\
\hline & GS3 & & G2019S Heterozygous \\
\hline & RG1 & & R1441G Heterozygous \\
\hline \multirow[t]{2}{*}{$R G$} & RG2 & 1931-1942 & R1441G Heterozygous \\
\hline & RG3 & & R1441G Heterozygous \\
\hline
\end{tabular}

The control group consists of four individuals, the IPD, GS and RG groups of three individuals each. We present in this table the range age of each group, and the genotype of each individual. 


\section{Protein Identification}

MS/MS data sets were identified using Mascot licensed version 2.3.02 (Matrix Sciences) and ProteinPilot (revision 4895; AB SCIEX 5.0.1) using the Paragon algorithm (5.0.1.0, 4874). All data files were searched using the SwissProtHuman 2015_09_17 database with 42136 sequences. Search parameters in Mascot for acetylated peptides were as follows: trypsin digestion with five missed cleavages to account the inability of trypsin to cleave at acetylated lysine residues. Lysine acetylation, Nterminal acetylation and methionine oxidation were set as variable modifications, and carbamidomethyl cysteine as a fixed modification. Precursor ion and fragment ion mass tolerances were set to $30 \mathrm{ppm}$ and $0.6 \mathrm{Da}$, respectively. Further, the decoy database search (Mascot integrated decoy approach) was used to false decoy recovery (FDR) calculation and the percolator algorithm applied to Mascot results. The acceptances criteria for proteins identification were a FDR $<1 \%$ and at least one peptide identified with a confidence interval (CI > 95\%). In ProteinPilot, the following sample parameters were used: trypsin digestion, cysteine alkylation with iodoacetamide, and acetylation emphasis. A thorough identification (ID) search was done. Thus, a local FDR of $1 \%$ was chosen using the ProteinPilot FDR analysis tool (PSPEP) algorithm and a peptide CI value of $95 \%$.

\section{Protein Relative Quantification}

For human proteins relative quantification in (Co, IPD, GS, and RG) samples, the Raw profile data files (.raw) were imported into Progenesis LC-MS for proteomics (64-bit version v 4.1; Nonlinear Dynamics/Waters). Imported runs were chromatographic aligned to the reference run identified by the software. All runs were selected for peak picking with the automatic sensitivity method (default settings) and filtered to include only peaks with a charge state between 2 and 5. All detected features were normalized against the reference run by Progenesis LC-MS. Between-subject comparison was used as experimental design (Co, IPD, GS, and RG). Spectral data from selected features $(p$-value $<0.05)$ were transformed to Mascot generic format (MGF) files with Progenesis LC-MS and exported for peptide/protein identification to Mascot search engine, using the searched parameters above described. Mascot search results, that exceed the acceptance criteria for identification (FDR $<$ $1 \%$, peptides with individual ion scores $>13, p<0.05$ ), were imported into Progenesis LC-MS as XML files and analyzed according to the following criteria: only were used quantitation from non-conflicting peptides. For each protein, the number or reported peptides was determined by counting unique peptide sequence. Only proteins reported by one or more peptides with a $p$-value $<0.05$ were quantified.

Protein abundance was calculated from the sum of all unique normalized peptide ion abundance. Protein reported abundance is the geometric mean of the biological replicates. Proteins with a likelihood of quantification smaller than 0.05 (Anova $p$-value) were considered to be significantly regulated. Normalized peptide intensities were used to calculate fold-changes between samples. Relative abundance of human proteins (fold change) in three conditions compared with corresponding proteins in control samples were quantified by the ratio of summed peptide ion normalized abundance in each group to evaluate the enrichment of the protein. Differentially expressed proteins $(p<0.05)$ were considered with a fold change $\geq 1.3$ and at least 1 identified peptides in at least one of replicates.

\section{Immunofluorescence}

HFs were seeded on 96-well plate at a density of 3500 cells/well. Cells were successively fixed with 4\% PFA and permeabilized with $0.1 \%$ Triton (Sigma-Aldrich, T9284) for 20 and $5 \mathrm{~min}$, respectively, at room temperature (RT). Once permeabilized, plated cells were incubated with bovine serum albumin (BSA)/PBS solution ( $1 \mathrm{mg} / \mathrm{mL}$ ) for $1 \mathrm{~h}$ at $\mathrm{RT}$ and then with the primary antibody acetyl-H4 (G-2) (Ser1K5K8K12) (1:50, Santa Cruz Biotechnology, sc-393472), while shaking overnight at $4^{\circ} \mathrm{C}$. The following day, cells were reincubated with Alexa Fluor ${ }^{\circledR} 568$ (1:100 Thermo Scientific, A11004)conjugated secondary antibodies for $1 \mathrm{~h}$ at RT. Nuclei were stained with Hoechst 33342 (2 $\mu$ M, Sigma Aldrich, B2261). Images were visualized using an Olympus IX51 inverted microscope.

\section{Statistical Analyses}

Statistical analyses were assessed by Student's $t$-test, Chi-Squared test or Anova test. The results were considered significant at $p<0.05$.

\section{RESULTS}

\section{Detection of Acetylated Proteins}

We determined the N-terminal acetylation and acetylated lysine (Ac-K) proteins in fibroblasts from PD patients and control subjects. In Tables 2, 3, we have listed some of those acetylated proteins. Some of them (fructose bisphosphate aldolase C (ALDOC), Glyceraldehyde-3-phosphate dehydrogenase (GAPDH), Alpha-Enolase, pyruvate kinase) are enzymes that participate at different steps of glycolysis pathway (TeSlaa and Teitell, 2014). The rest of acetylated proteins are implicated in cell proliferation, acetylation, apoptosis and nucleosome wrap, as well in the link between glycolysis and citric acid cycle. Most of those proteins were located in the nucleus and the cytoplasm and represent, respectively, 52 and $36 \%$ of acetylated proteins in PD patients (Figure 1A). The less represented were found in plasma membrane (8\%) and mitochondrion (4\%). This distribution did not change in the control group but the proportion slightly differs. The number of Ac-K sites on proteins varied from one to ten (Tables 2, 3) and can be found on distinct peptides. Furthermore, in PD models some acetylated proteins had more or less Ac-K sites than Control line (Table 2). Interestingly, we found that the ratio of acetylated peptides/non-acetylated peptides was enhanced in GS and RG cells while it was decreased in IPD cells (Figure 1B). We inferred that there were more acetylated peptides levels in familial PD than in sporadic PD. These variations were significant between genetic and idiopathic PD. 
TABLE 2 | Acetylated proteins in fibroblasts from PD patients with or without LRRK2 mutation.

\begin{tabular}{|c|c|c|c|}
\hline Protein ID & Protein Name & Subcellular Location & Ac-K Sites \\
\hline P07355 & Annexin A2 & $\begin{array}{l}\text { Extracellular space, Extracellular } \\
\text { matrix }\end{array}$ & 10 \\
\hline P04083 & Annexin A1 & $\begin{array}{l}\text { Nucleus, Cytoplasm, Cell } \\
\text { membrane }\end{array}$ & 1 \\
\hline P08670 & Vimentin & Cytoplasm & 2 \\
\hline Q9BQE3 & $\alpha$-Tubulin $1 C$ & Cytoplasm, Cytoskeleton & 1 \\
\hline P68363 & $\alpha$-Tubulin 1B & Cytoplasm, Cytoskeleton & 1 \\
\hline P14618 & Pyruvate kinase & Cytoplasm, Nucleus & 2 \\
\hline Q6PEY2 & $\alpha$-Tubulin 3E & Cytoplasm, Cytoskeleton. & 1 \\
\hline P08758 & Annexin A5 & - & 4 \\
\hline P21333 & Filamin-A & $\begin{array}{l}\text { Cytoplasm, Cell cortex, } \\
\text { Cytoskeleton. }\end{array}$ & 1 \\
\hline P62805 & Histone H4 & Nucleus, Chromosome & 4 \\
\hline Q9GZZ1 & NAA50 & Cytoplasm & 2 \\
\hline Q09472 & HAT p300 & Cytoplasm, Nucleus. & 5 \\
\hline Q92793 & $\mathrm{CBP}$ & Cytoplasm, Nucleus. & 2 \\
\hline Q99880 & H2B1L & Nucleus, Chromosome & 4 \\
\hline P04406 & GAPDH & Cytoplasm, Cytoskeleton & 4 \\
\hline P68371 & $\beta$-Tubulin 4B & $\begin{array}{l}\text { Cytoplasm, Cytoskeleton, } \\
\text { Nucleus }\end{array}$ & 1 \\
\hline P07437 & $\beta$-Tubulin & Cytoplasm, Cytoskeleton. & 1 \\
\hline P06733 & Alpha-enolase & Cytoplasm, Cell membrane & 4 \\
\hline O43809 & CPSF5 & Nucleus & 1 \\
\hline P62328 & Thymosin $\beta-4$ & Cytoplasm, Cytoskeleton. & 3 \\
\hline P22392 & NME2 & Cytoplasm, Nucleus & 2 \\
\hline Q15942 & Zyxin & $\begin{array}{l}\text { Cytoplasm, Cytoskeleton, } \\
\text { Nucleus, Cell junction }\end{array}$ & 2 \\
\hline P21796 & VDAC1 & Outer mitochondrial membrane & 1 \\
\hline P20962 & Parathymosin & Nucleus & 4 \\
\hline P08559 & PDHA1 & Mitochondrial matrix & 4 \\
\hline P06454 & Prothymosin $\alpha$ & Nucleus & 5 \\
\hline P68871 & Hemoglobin $\beta$ & - & 1 \\
\hline P02042 & Hemoglobin $\Delta$ & - & 1 \\
\hline P09972 & ALDOC & - & 1 \\
\hline P04792 & $\begin{array}{l}\text { Heat shock protein } \\
\text { beta- } 1\end{array}$ & Cytoplasm, Nucleus & 1 \\
\hline P06703 & Protein S100-A6 & Nucleus envelope, Cell membrane & 1 \\
\hline P00338 & LDHA A & Cytoplasm & 4 \\
\hline
\end{tabular}

We specified the subcellular location of proteins and the number of acetylation sites. Lines filled in blue represent the most relevant variations identified in PD models compared to control.

\section{Identification of Acetylated Peptides and Proteins in PD Fibroblasts}

To elucidate these differences, PD patients' data were compared to Co data. We observed that there were four acetylated proteins only belonged to Co (Figures 1C-E), however two of them (Nucleophosmin and Zinc finger protein 784) were constant. The pairs of thymosin $\beta 4$ and $\mathrm{H} 2 \mathrm{~B} 1 \mathrm{~B}$ or $\mathrm{H} 2 \mathrm{~B} 1 \mathrm{~B}$ and $\mathrm{H} 2 \mathrm{~B} 1 \mathrm{M}$ or Annexin $\mathrm{A} 6$ and Histone 1.5 were additionnally found in Co line when compared to IPD, GS, and RG lines, respectively. Moreover, Co and PD lines
TABLE 3 | Acetylated proteins in human fibroblasts from control subjects.

\begin{tabular}{|c|c|c|c|}
\hline Protein ID & Protein Name & Subecellular Location & Ac-K Sites \\
\hline P07355 & Annexin A2 & $\begin{array}{l}\text { Extracellular space, } \\
\text { Extracellular matrix }\end{array}$ & 10 \\
\hline P04083 & Annexin A1 & $\begin{array}{l}\text { Nucleus, Cytoplasm, Cell } \\
\text { membrane }\end{array}$ & 2 \\
\hline P08670 & Vimentin & Cytoplasm & 4 \\
\hline Q9BQE3 & Tubulin $\alpha-1 C$ & Cytoplasm, Cytoskeleton & 2 \\
\hline P08758 & Annexin A5 & - & 3 \\
\hline P21333 & Filamin-A & $\begin{array}{l}\text { Cytoplasm, Cell cortex, } \\
\text { Cytoskeleton. }\end{array}$ & 1 \\
\hline P62805 & Histone $\mathrm{H} 4$ & Nucleus, Chromosome & 3 \\
\hline Q9GZZ1 & NAA50 & Cytoplasm & 2 \\
\hline Q09472 & HAT p300 & Cytoplasm, Nucleus. & 1 \\
\hline Q92793 & $\mathrm{CBP}$ & Cytoplasm, Nucleus. & 2 \\
\hline Q99880 & H2B1L & Nucleus, Chromosome & $4-7$ \\
\hline P04406 & GAPDH & Cytoplasm, Cytoskeleton & 2 \\
\hline P68371 & Tubulin $\beta-4 B$ & $\begin{array}{l}\text { Cytoplasm, Cytoskeleton, } \\
\text { Nucleus }\end{array}$ & 1 \\
\hline P07437 & Tubulin $\beta$ & Cytoplasm, Cytoskeleton. & 1 \\
\hline P06733 & Alpha-enolase & Cytoplasm, Cell membrane & 4 \\
\hline O43809 & CPSF5 & Nucleus & 1 \\
\hline P62328 & Thymosin $\beta-4$ & Cytoplasm, Cytoskeleton. & 2 \\
\hline P22392 & NME2 & Cytoplasm, Nucleus & 2 \\
\hline Q15942 & Zyxin & $\begin{array}{l}\text { Cytoplasm, Cytoskeleton, } \\
\text { Nucleus, Cell junction }\end{array}$ & 2 \\
\hline P21796 & VDAC1 & $\begin{array}{l}\text { Outer mitochondrial } \\
\text { membrane }\end{array}$ & 1 \\
\hline P20962 & Parathymosin & Nucleus & 2 \\
\hline P06454 & Prothymosin $\alpha$ & Nucleus & 4 \\
\hline P68871 & Hemoglobin $\beta$ & - & 1 \\
\hline P02042 & Hemoglobin $\Delta$ & - & 1 \\
\hline P09972 & ALDOC & - & 1 \\
\hline P06703 & Protein S100-A6 & $\begin{array}{l}\text { Nucleus envelope, Cell } \\
\text { membrane }\end{array}$ & 1 \\
\hline P00338 & LDHA A & Cytoplasm & 4 \\
\hline P04075 & ALDOA & - & 1 \\
\hline P08133 & ANXA6 & Cytoplasm & 4 \\
\hline P06748 & NPM & Cytoplasm, Nucleus & 1 \\
\hline Q8NCA9 & ZN784 & Nucleus & 1 \\
\hline P33778 & H2B1B & Nucleus & 3 \\
\hline P16401 & Histone1.5 & Nucleus & 2 \\
\hline
\end{tabular}

The subcellular location of proteins and the number of $A c-k$ sites.

have acetylated proteins in common. From the detected acetylated proteins based on the comparison effectuated respect to Co line, distinct acetylated peptides were identified and were characteristic of each group. Thus, 2 out of 5 acetylated peptides [STVHEILCK (Annexin A2 protein) and KGSKKAVTKAQKK (H2B1L protein)] are specific to IPD line, 2 to GS line [FLEQQNKILLAELEQLK (Vimentin protein) and KGSKKAVTK (H2B1C protein)] and 1 out of 3 to RG line [GVTQFGNKYIQQTK (CPSF5 protein)]. Of note, proteins can be acetylated in one or more peptides, and following the group of healthy subjects or PD patients, the acetylation of 

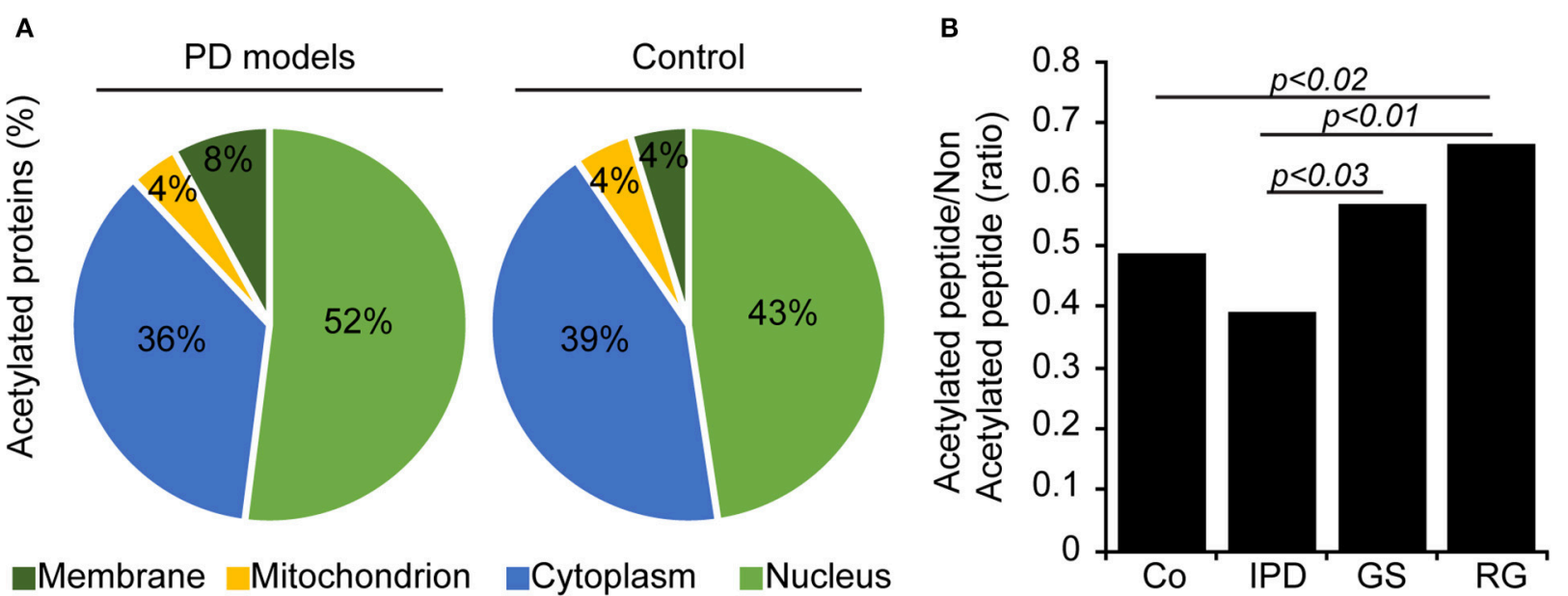

C
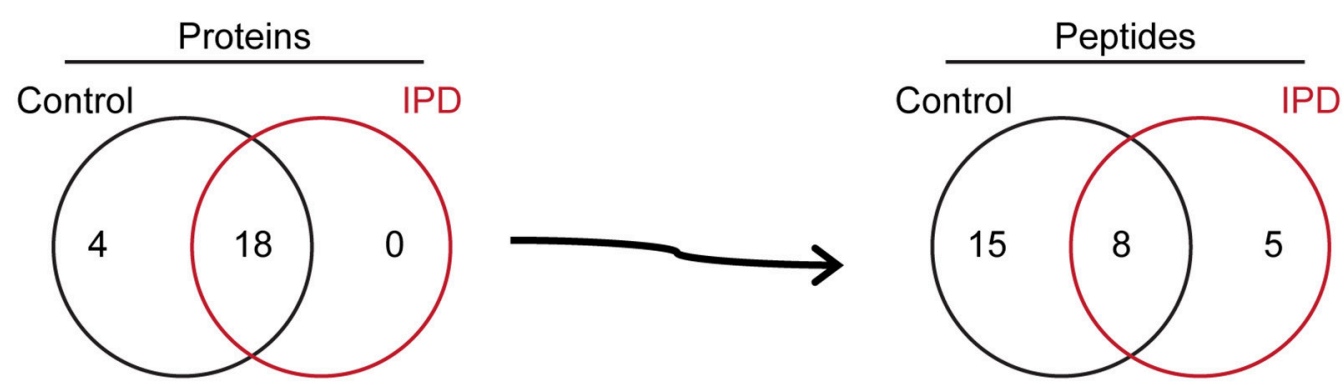

D
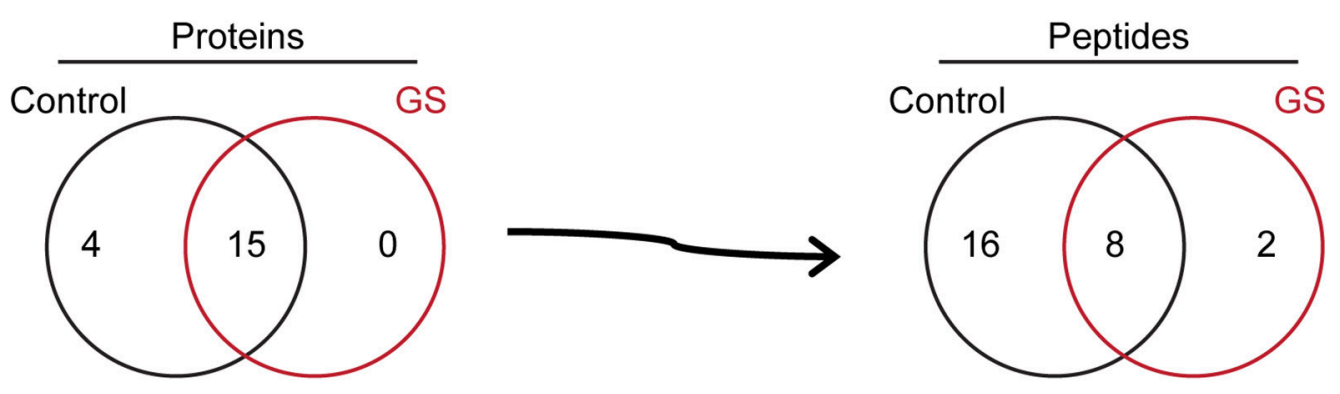

E
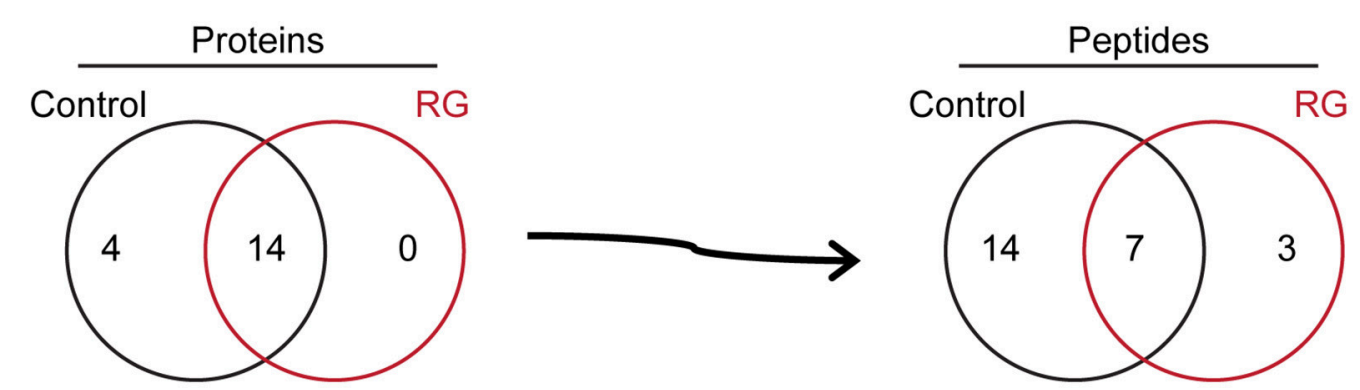

FIGURE 1 | Acetylated proteins in human fibroblasts. (A) Subcellular location of acetylated proteins (\%) in human fibroblasts. (B) Ratio of identified acetylated peptides/non-acetylated peptides in human fibroblasts, $p<0.03, p<0.02$ and $p<0.01$ compared to control ( $\chi^{2}$-test). (C-E) Comparison of acetylated proteins and identified acetylated peptides per proteins between Co line and PD models. (C) Co and IPD lines, (D) Co and GS lines (E) Co and RG lines represent the acetylome of each group and what they share in common. 
one protein might change from one peptide to another and be monoactylated, polyacetylated or inexistent. Given that the fold change of acetylated peptides depend on the relative abundance of proteins, the level of acetylated peptides was classified in three categories (Normal, hypoacetylated, and hyperacetylated). Therefore, it exists more hypoacetylated peptides in IPD line than in Co line. Also, the percentage of hyperacetylated peptides was significantly increased in cells harboring LRRK2 mutations (Figure 2A). By immunofluorescence staining, we observed that the intensity of acetylated Histone 4 was significantly reduced in IPD line (Figures 2B,C).

\section{DISCUSSION}

Dysregulation of acetylation machinery can lead to neurodegenerative diseases. In fact, protein aggregation is a hallmark of neurodegeneration and it is regulated by protein acetylation including lysine acetylation (Lee and Finkel, 2009) and $\mathrm{N}$-terminal acetylation. It has been reported that the $\mathrm{N}$-terminal acetylation of SNCA prevents its aggregation by stabilizing protein formation (Bartels et al., 2014), besides that, N-terminal acetylation can also act as a recognition tag to mediate protein degradation (Zattas et al., 2013), thus Nacetylation deficiency could be associated with PD pathogenesis. Despite this protective effect, $\mathrm{N}$-terminal acetylation can disturb further proteasome degradation interplaying the $\mathrm{N}$-terminal ubiquitylation of $\alpha$-amino group of proteins substrates (Tatham et al., 2013).

Even though $80 \%$ of human proteins are acetylated at their $\mathrm{N}$-terminal, they are poorly considered (Aksnes et al., 2016). In this study, the proteins N-terminally acetylated in PD models are annexin A2, putative annexin A2-like protein, cytosolic (Thymosin $\beta 4, \alpha$-enolase), nuclear (parathymosin, protein S100A6, prothymosin $\alpha$ ), and participate in various cellular processes. These proteins are more represented in IPD line. In common to IPD and GS lines, prothymosin $\alpha$ (Qi et al., 2010) and
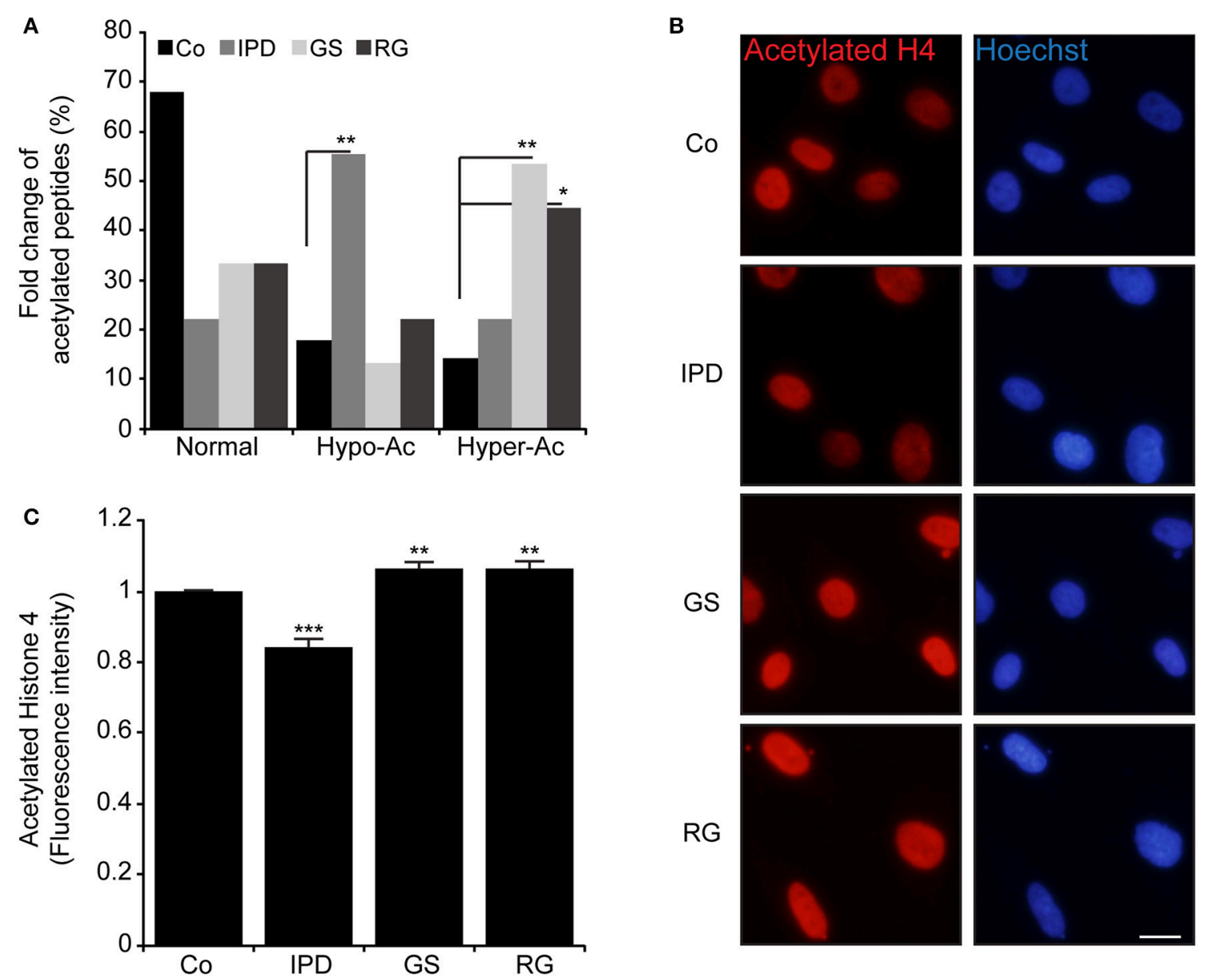

FIGURE 2 | Modulation of acetylated peptides in human fibroblast (A) Represents in \% the fold change of hypoacetylated and hyperacetylated peptides in HFs compared to Co, ${ }^{*} p<0.05$ and ${ }^{* *} p<0.01$ ( $\chi^{2}$-test). (B,C). Acetylated histone 4 (Ac-H4K5K8K12) (red) was detected by immunofluorescence and the nuclei were stained with Hoechst 33342 (blue), Original magnification: 20X, scale bar corresponds to $10 \mu \mathrm{m}$. (C) Represents the quantification of fluorescence intensity of labeled Ac-H4K5K8K12 by imageJ ( $n=200$ cells). Data represent the mean \pm SEM of at least three independent experiments, ${ }^{* *} p<0.01$ and ${ }^{* * *} p<0.001$ respect to Co (Student's t-test). 
protein S100-A6 (Bartkowska et al., 2017) are, respectively, antiapoptotic and stress modulator. $\alpha$-Enolase is found in RG line whereas annexin A2 is in all PD models. Annexins (A1, A2, and A5) are calcium sensors that translocate to plasma or nuclear membrane (Skrahina et al., 2008) and affect apoptosis pathways (Debret et al., 2003; Jiang et al., 2015). It remains to investigate whether the $\mathrm{N}$-acetylated form of these proteins have a crucial role in the progression of PD. Acetylated thymosin $\beta 4$ is common to both genetic PD lines, this protein has a critical role in actin polymerization (Mannherz et al., 2010). Moreover, LRRK2 mutations influence cytoskeleton organization. In fact, GTP LRRK2 domain interacts with $\beta$-tubulin and increases the lysine acetylation of $\alpha$-tubulin. However, this interaction is altered with G2019S and R1441G LRRK2 mutations and affects the stability of microtubules (Law et al., 2014). Indeed, neither microtubule structure nor conformation has affected by $\alpha$-tubulin acetylation but influences the tubulin-binding proteins therefore tubulin functions (Howes et al., 2014). Damaged mitochondria-induced reactive oxygen species (ROS) are responsible for $\alpha$-tubulin hyperacetylation (Bonet-Ponce et al., 2016). Such modification is required for an adaptive cell response through autophagy induction, consequently, this hyperacetylation is negatively regulated by p300 upon stress (Mackeh et al., 2014). In G2019S LRRK2 mutation, the reduction of mitochondrial membrane potential is accompanied by an increase of ROS production and an enhancement of the basal autophagy level (Yakhine-Diop et al., 2014). An impairment of autophagy induction was also observed in IPD and R1441G LRRK2 lines (Data not shown). The HATs p300 and CBP are acetylated in $\mathrm{RG}$ line, respectively, at five and 2 lysine positions, which means p300 basal activity is increased (Drazic et al., 2016). Even though, HAT and HDAC are involved in the modulation of protein acetylation, acetyl-CoA availability is critical. In mammals, acetyl CoA is in part generated from pyruvate by pyruvate dehydrogenase (PDHA1). Pyruvate is the final product of glycolysis pathway (Drazic et al., 2016). Some enzymes of this pathway are lysine acetylated in IPD, GS, and RG lines. This posttranslational modification can increase or decrease the enzymatic activity of certain proteins. In the case of acetylated PDHA1 in RG line, its activity is decreased (Drazic et al., 2016), therefore the level of acetyl-CoA formation from glycolysis may be reduced. The different pathways (glycolysis and fatty acid $\beta$-oxidation) generating acetyl CoA interplay in mitochondria. Generally, mitochondria are defective in PD, this dysfunction disturbs the acetylation machinery by reducing HDAC Class III (sirtuins) activity (Schwab et al., 2017). Additionally, an imbalance between HDAC and HAT activities lead to hyperacetylation (Park et al., 2016) or hypoacetylation

\section{REFERENCES}

Aksnes, H., Drazic, A., Marie, M., and Arnesen, T. (2016). First things first: vital protein marks by N-terminal acetyltransferases. Trends Biochem. Sci. 41, 746-760. doi: 10.1016/j.tibs.2016.07.005

Ammal Kaidery, N., Tarannum, S., and Thomas, B. (2013). Epigenetic landscape of Parkinson's disease: emerging role in disease mechanisms and therapeutic modalities. Neurotherapeutics 10, 698-708. doi: 10.1007/s13311-013-0211-8 of proteins. Taken together, proteins are acetylated in IPD and LRRK2 mutations-associated PD. However, it occurs more hyperacetylated proteins in cells harboring LRRK2 mutations than in IPD lines. This variation seems to be associated with the disease. Moreover, in healthy subjects harboring the R1441G LRRK2 mutation, the intensity of acetylated proteins was enhanced (Data not shown). The molecular mechanism of protein acetylation in PD remains unclear. It will be interesting to elucidate how proteins can be hypoacetylated in IPD rather than in Genetic PD.

\section{AUTHOR CONTRIBUTIONS}

JF conceived the project. SY-D, JB-S, RG-S, MN-S, RG-P, and JF designed the experiments. SY-D, MR-A, GM-C, and EU-C performed experiments. MR-A performed the statistical analyses. Authors assisted in data analysis and interpretation. AA and AL performed the human fibroblast biopsies and culture. SY-D and JF wrote the manuscript. All authors revised and approved the content of the manuscript for publication.

\section{ACKNOWLEDGMENTS}

We are grateful to the patients and donors without which these work would not have been possible. The authors thank M. P. Delgado-Luceño. The proteomic analysis was performed in the Proteomics Facility of UCM that belongs to ProteoRed, PRB2-ISCIII, supported by grant PT13/0001. SY-D was supported by Isabel Gemio Foundation. EU-C was supported by a FPU predoctoral fellowship (FPU16/00684) from Ministerio de Educación, Cultura y Deporte, Spain. RG$S$ was supported by a Marie Sklodowska-Curie Individual Fellowship (IF-EF) (655027) from the European Commission. JB-S was funded by La Ligue Contre le Cancer. MN-S was supported by Contrato Ramon y Cajal (RYC-2016-20883) from Ministerio de Economia y Competitividad, Spain. MR-A was supported by a FPU predoctoral fellowship (FPU13/01237) from Ministerio de Educación, Cultura y Deporte, Spain. JF received research support from the Instituto de Salud Carlos III, CIBERNED (CB06/05/004) and Instituto de Salud Carlos III, FIS (PI15/00034). RG-P was supported by a Contrato destinado a la retención y atracción del talento investigador, TA13009 from Junta de Extremadura, as well as research support from the Instituto de Salud Carlos III, FIS (PI14/00170). This work was also supported by Fondo Europeo de Desarrollo Regional (FEDER) from the European Union. The authors also thank FUNDESALUD for helpful assistance.

Bartels, T., Kim, N. C., Luth, E. S., and Selkoe, D. J. (2014). N-alphaacetylation of alpha-synuclein increases its helical folding propensity, GM1 binding specificity and resistance to aggregation. PLOS ONE 9:e103727. doi: 10.1371/journal.pone.0103727

Bartkowska, K., Swiatek, I., Aniszewska, A., Jurewicz, E., Turlejski, K., Djavadian, A., et al. (2017). Stress-dependent changes in the CacyBP/SIP interacting protein S100A6 in the mouse brain. PLoS ONE 12:e0169760. doi: 10.1371/journal.pone.0169760 
Bonet-Ponce, L., Saez-Atienzar, S., da Casa, C., Sancho-Pelluz, J., Barcia, J. M., Martinez-Gil, N., et al. (2016). Rotenone induces the formation of 4-hydroxynonenal aggresomes. role of ROS-mediated tubulin hyperacetylation and autophagic flux disruption. Mol. Neurobiol. 53, 6194-6208. doi: 10.1007/s12035-015-9509-3

Coppedè, F. (2012). Genetics and epigenetics of Parkinson's disease. Sci. World J. 2012:489830. doi: 10.1100/2012/489830

Debret, R., El Btaouri, H., Duca, L., Rahman, I., Radke, S., Antonicelli, B., et al. (2003). Annexin Al processing is associated with caspase-dependent apoptosis in BZR cells. FEBS Lett. 546, 195-202. doi: 10.1016/S0014-5793(03)00570-2

Deusser, J., Schmidt, S., Ettle, B., Plotz, S., Huber, S., Kohl, C. P., et al. (2015). Serotonergic dysfunction in the A53T $\alpha$-synuclein mouse model of Parkinson's disease. J. Neurochem. 135, 589-597. doi: 10.1111/jnc.13253

Drazic, A., Myklebust, L. M., Ree, R., and Arnesen, T. (2016). The world of protein acetylation. Biochim. Biophys. Acta 1864, 1372-1401. doi: 10.1016/j.bbapap.2016.06.007

Howes, S. C., Alushin, G. M., Shida, T., Nachury, M. V., and Nogales, E. (2014). Effects of tubulin acetylation and tubulin acetyltransferase binding on microtubule structure. Mol. Biol. Cell 25, 257-266. doi: 10.1091/mbc.E13-07-0387

Jiang, S. L., Pan, D. Y., Gu, C., Qin, H. F., and Zhao, S. H. (2015). Annexin A2 silencing enhances apoptosis of human umbilical vein endothelial cells in vitro. Asian Pac. J. Trop. Med. 8, 952-957. doi: 10.1016/j.apjtm.2015. 10.006

Law, B. M., Spain, V. A., Leinster, V. H., Chia, R., Beilina, A., Harvey, H. J., et al. (2014). A direct interaction between leucine-rich repeat kinase 2 and specific $\beta$-tubulin isoforms regulates tubulin acetylation. J. Biol. Chem. 289, 895-908. doi: 10.1074/jbc.M113.507913

Lee, I. H., and Finkel, T. (2009). Regulation of autophagy by the p300 acetyltransferase. J. Biol. Chem. 284, 6322-6328. doi: 10.1074/jbc.M807135200

Li, J. Q., Tan, L., and Yu, J. T. (2014). The role of the LRRK2 gene in Parkinsonism. Mol. Neurodegener. 9:47. doi: 10.1186/1750-1326-9-47

Mackeh, R., Lorin, S., Ratier, A., Mejdoubi-Charef, N., Baillet, A., Perdiz, A., et al. (2014). Reactive oxygen species, AMP-activated protein kinase, and the transcription cofactor p300 regulate $\alpha$-tubulin acetyltransferase1 ( $\alpha$ TAT-1/MEC-17)-dependent microtubule hyperacetylation during cell stress. J. Biol. Chem. 289, 11816-11828. doi: 10.1074/jbc.M113.5 07400

Mannherz, H. G., Mazur, A. J., and Jockusch, B. (2010). Repolymerization of actin from actin:thymosin $\beta 4$ complex induced by diaphanous related formins and gelsolin. Ann. N. Y. Acad. Sci. 1194, 36-43. doi: 10.1111/j.1749-6632.2010.05467.x

Martin, I., Kim, J. W., Dawson, V. L., and Dawson, T. M. (2014). LRRK2 pathobiology in Parkinson's disease. J. Neurochem. 131, 554-565. doi: $10.1111 /$ jnc. 12949

Park, G., Tan, J., Garcia, G., Kang, Y., Salvesen, G., and Zhang, Z. (2016). Regulation of histone acetylation by autophagy in Parkinson Disease. J. Biol. Chem. 291, 3531-3540. doi: 10.1074/jbc.M115. 675488
Politis, M., and Loane, C. (2011). Serotonergic dysfunction in Parkinson's disease and its relevance to disability. Sci. World J. 11, 1726-1734. doi: 10.1100/2011/172893

Qi, X., Wang, L., and Du, F. (2010). Novel small molecules relieve prothymosin $\alpha$ mediated inhibition of apoptosome formation by blocking its interaction with Apaf-1. Biochemistry 49, 1923-1930. doi: 10.1021/bi9022329

Schwab, A. J., Sison, S. L., Meade, M. R., Broniowska, K. A., Corbett, J. A. and Ebert, A. D. (2017). Decreased sirtuin deacetylase activity in LRRK2 G2019S iPSC-derived dopaminergic neurons. Stem Cell Reports 9, 1839-1852. doi: 10.1016/j.stemcr.2017.10.010

Skrahina, T., Piljić, A., and Schultz, C. (2008). Heterogeneity and timing of translocation and membrane-mediated assembly of different annexins. Exp. Cell Res. 314, 1039-1047. doi: 10.1016/j.yexcr.2007.11.015

Song, C., Kanthasamy, A., Jin, H., Anantharam, V., and Kanthasamy, A. G. (2011). Paraquat induces epigenetic changes by promoting histone acetylation in cell culture models of dopaminergic degeneration. Neurotoxicology 32, 586-595. doi: 10.1016/j.neuro.2011.05.018

Song, C., Kanthasamy, A., Anantharam, V., Sun, F., and Kanthasamy, A. G. (2010). Environmental neurotoxic pesticide increases histone acetylation to promote apoptosis in dopaminergic neuronal cells: relevance to epigenetic mechanisms of neurodegeneration. Mol. Pharmacol. 77, 621-632. doi: $10.1124 / \mathrm{mol} .109 .062174$

Tatham, M. H., Plechanovova, A., Jaffray, E. G., Salmen, H., and Hay, R. T. (2013). Ube $2 \mathrm{~W}$ conjugates ubiquitin to $\alpha$-amino groups of protein $\mathrm{N}$-termini. Biochem. J. 453, 137-145. doi: 10.1042/BJ20130244

TeSlaa, T., and Teitell, M. A. (2014). Techniques to monitor glycolysis. Methods Enzymol. 542, 191-114. doi: 10.1016/B978-0-12-416618-9.00005-4

Yakhine-Diop, S. M., Bravo-San Pedro, J. M., Gómez-Sánchez, R., RodríguezArribas, E., Rodriguez-Arribas, M., Gonzalez-Polo, V., et al. (2014). G2019S LRRK2 mutant fibroblasts from Parkinson's disease patients show increased sensitivity to neurotoxin 1-methyl-4-phenylpyridinium dependent of autophagy. Toxicology 324, 1-9. doi: 10.1016/j.tox.2014.07.001

Zattas, D., Adle, D. J., Rubenstein, E. M., and Hochstrasser, M. (2013). $\mathrm{N}$-terminal acetylation of the yeast Derlin Der1 is essential for Hrd1 ubiquitinligase activity toward luminal ER substrates. Mol. Biol. Cell 24, 890-900. doi: 10.1091/mbc.E12-11-0838

Conflict of Interest Statement: The authors declare that the research was conducted in the absence of any commercial or financial relationships that could be construed as a potential conflict of interest.

Copyright (C) 2018 Yakhine-Diop, Rodríguez-Arribas, Martínez-Chacón, UribeCarretero, Gómez-Sánchez, Aiastui, López de Munain, Bravo-San Pedro, NisoSantano, González-Polo and Fuentes. This is an open-access article distributed under the terms of the Creative Commons Attribution License (CC BY). The use distribution or reproduction in other forums is permitted, provided the original author(s) and the copyright owner are credited and that the original publication in this journal is cited, in accordance with accepted academic practice. No use, distribution or reproduction is permitted which does not comply with these terms. 